\title{
TOT en el tratamiento de la incontinencia de esfuerzo: nuestra experiencia, comparándola con la TVT
}

\author{
Jiménez Calvo J, Hualde Alfaro A, De Pablo Cárdenas A, Cebrian Lostal JL, Álvarez Bandres S, \\ Raigoso Ortega O.
}

Servicio de Urología. Hospital Virgen del Camino. Pamplona. Navarra.

Actas Urol Esp. 2007;31(10):1134-1140

\section{RESUMEN \\ TOT EN EL TRATAMIENTO DE LA INCONTINENCIA DE ESFUERZO: NUESTRA EXPERIENCIA, COMPARANDOLA CON LA TVT}

Introducción: En el año 2004 iniciamos la técnica de TOT para el tratamiento de la incontinencia urinaria de esfuerzo. En este estudio comparamos los resultados con las series publicadas de TOT y con la serie de TVT realizadas en nuestro servicio.

Material y métodos: Hemos intervenido a 171 pacientes, con una edad media de 57,7 años. En el $167 \%$ de ellas, además de realizar TOT, asociamos reparación de defectos anatómicos pélvicos.

Resultados: Con un seguimiento medio de 12 meses y mediana de 14 meses, el 87\% de las pacientes están curadas. Los fracasos aparecieron de forma precoz en los 3 primeros meses de seguimiento. Como complicaciones aparecieron, retenciones post-operatorias de menos de 30 días en el 2,7\%, retenciones a largo en el $4,8 \%$, hematomas post-quirúrgicos en el $1,3 \%$, extrusión de la malla en el $2 \%$ y urgencia de novo en el $2.3 \%$. Conclusiones: La TOT es una técnica no exenta de complicaciones aunque la proporción de ellas es baja y con unos resultados que son alentadores. Se trata de una técnica quirúrgica sencilla, que como toda técnica, tiene una curva de aprendizaje. Requiere un corto tiempo quirúrgico (menor que la TVT) y puede ser realizada en régimen de Cirugia Mayor Ambulatoria. Las dos técnicas (TVT/TOT) han demostrado ser similares en cuanto a tasas de curación aunque en la serie de TOT es levemente más baja. Las diferencias de las complicaciones se dan en los porcentajes aunque siempre escasas.

Palabras clave: TVT. TOT. Incontinencia de esfuerzo.

\section{ABSTRACT \\ TOT IN THE TREATMENT OF THE STRESS URINARY INCONTINENCE: OUR EXPERIENCE, COMPARING IT WITH THE TVT}

Introduction: In the year 2004 begin the technique of TOT for the treatment of the urinary incontinence of effort. In this study we compare the results with the published series of TOT and with the series of TVT carried out in our service.

Material and methods: We have intervened to 171 patients, with a 57.7 year-old half age. In their $16 \%$, besides carrying out TOT, we associate repair of anatomical pelvic defects.

Results: After a follow-up of 12 months and a mean of $14,87 \%$ of the cases succeeded. Failures arose during the first three months of therapy. As complications they appeared post-operative retentions of less than 30 days in $2.7 \%$, retentions to long in $4.8 \%$, post-surgical hematomas in $1.3 \%$, extrusion of the mesh in $2 \%$ and novo urgency in $2.3 \%$.

Conclusions: TOT is a technique it doesn't exempt of complications although their proportion is low and with some results that they are encouraging. It is about a surgical simple technique that as all technique, he/she has a learning curve. It requires a surgical (smaller than TVT) short time and it can be carried out in régime of bigger Ambulatory Surgery. The two techniques (TVT / TOT) have demonstrated to be similar as for cure rates although in the series of TOT it is slightly more baja. Las differences of the complications they are given in the percentages although always scarce.

Keywords: TVT. TOT. Stress urinary incontinence. 
El tratamiento de la incontinencia urinaria de esfuerzo (IUE) ha ido variando en el tiempo, y en este momento la porción suburetral de la uretra media es determinante en cualquier estrategia que busque su corrección quirúrgica en el tratamiento de la IUE.

El procedimiento denominado TVT para el tratamiento de la incontinencia urinaria de esfuerzo se basa en una nueva teoría sobre los mecanismos de la continencia en la mujer: la teoría integral, según la cual se cierra la uretra en la porción media. La falta de soporte en la uretra media por los ligamentos pubouretrales y el deterioro de la función e inserción de los músculos pubococcígeos provocan el descenso y apertura de la uretra predisponiendo a las pacientes a presentar $\mathrm{IUE}^{1,2}$.

Desde que U.Ulmsten y P.Petros describieron la técnica TVT por primera vez en 1996, los resultados preliminares han sido corroborados en publicaciones posteriores con un seguimiento más largo. Los buenos resultados, $84,6 \%$ de curaciones, se mantienen hasta los 60 meses de seguimiento $^{4,5}$.

Como variante para el emplazamiento del sling suburetral retropubiano, Delorme ideó el abordaje alternativo por vía obturatriz $(2001)^{6}$. El TOT (tension free trans obturator tape) consiste en colocar la malla suburetral pasándola, a cada lado, por el orificio obturador. Las ventajas son: evita la apertura del espacio de Retzius, disminuyendo la probabilidad de lesiones de víscera hueca, hemorragias y hematomas en dicho espacio y haciendo innecesaria la realización de cistoscopia intraoperatoria ya que la cinta se ubica en un plano inferior a la vejiga.

Partiendo de estas premisas analizamos nuestros resultados de la primeras 171 TOT colocadas en nuestro servicio y comparamos su eficacia con la TVT (de nuestra serie) así como las complicaciones precoces y tardías de ambas.

\section{MATERIAL Y MÉTODOS}

Entre Febrero de 2004 hasta Junio de 2006 hemos tratado a 171 pacientes con incontinencia urinaria, de las cuales 110 presentaban una incontinencia urinaria de esfuerzo pura y 61 incontinencia urinaria mixta. La edad media de las pacientes es de 57,7 años (rango entre 33-92 años). La mediana de seguimiento es de 14 meses y la media de 12 meses. La duración de los síntomas antes de consultar al especialista fue una mediana de 6.71 años con un rango entre 1 año y 27 años. El 20\% de las pacientes presentaban antecedentes de cirugía vaginal (histerectomía, plastia vaginal,) y el 3\% cirugía antiincontinencia.

Todas las pacientes fueron sometidas a que incluía la búsqueda de cistocele, rectocele, prolapso uterino, prueba de esfuerzo, test de Boney y función de los músculos pelvianos. A las pacientes se les preguntaba cuanto afectaba a su calidad de vida la incontinencia, teniendo en cuenta que 0 significaba no afectación de su calidad y 10 afectación importante, en una escala visual. Su media resultó de 7.18 y mediana de 8 lo que indica que su calidad de vida estaba francamente afectada.

Dentro del estudio preoperatorio se incluía cistomanometría fundamentalmente en aquellas pacientes que presentaban incontinencia mixta (el 80\% de las pacientes tienen urodinámica).En las que tenian IUE pura sólo se hizo urodinámica el $20 \%$ de ellas.

En la urodinámica realizada en las pacientes que presentaban IUE pura (21 pacientes) corroboramos la IUE, constatamos Presión de fuga menores a $80 \mathrm{~cm} \mathrm{H} 2 \mathrm{O}$ y vejigas estables en todas las pacientes excepto 3: una de ellas presentaba contracciones no inhibidas a $400 \mathrm{cc}$ sin escapes acompañantes, otra contracciones no inhibidas a 170 cc (antecedentes de TVT previa fracasada) y la última un detrusor hipoactivo posiblemente secundario a diabetes insulino dependiente de varios años de evolución.

La urodinámica realizada en las pacientes que presentaban IUMixta (48 pacientes) mostró en la mitad contracciones no inhibidas cuyo volumen de aparición variaba entre 50 cc y 449 cc, y en la otra mitad se objetivaba IUE pero con vejigas estables.

Consideramos como incontinencias de minimos esfuerzos aquellas que se producen al andar; moderadas aquellas que tienen lugar cuando tosen o ríen y de grandes esfuerzos cuando aparecen al coger pesos importantes o aparecen al hacer ejercicio físico (Tabla 1), aunque reconocemos que esta clasificación es arbi- 
Tabla 1. Tipos de incontinencia de los 171 pacientes

\begin{tabular}{lc}
\hline Grandes esfuerzos & $6 \%$ \\
Moderados esfuerzos & $35 \%$ \\
Minimos esfuerzos & $24 \%$ \\
Incontinencia Mixta & $35 \%$ \\
\hline
\end{tabular}

traria y subjetiva, nos da una idea del grado de incontinencia y no utilizamos el "pad test" ya que creemos que es también subjetiva su valoración y mucho más laboriosa.

Dentro de la exploración física damos mucha importancia a la valoración del cistocele, ya que según su cuantificación tendrá implicaciones quirúrgicas. Consideramos cistocele grado I cuando no alcanza el introito, grado II cuando éste llega hasta introito vaginal y grado III cuando pasa más allá del introito vaginal (Tabla 2$)^{3}$.

Tabla 2. Grados de cistocele intervenido de los 171 pacientes

\begin{tabular}{lc}
\hline Cistocele grado O & $9 \%$ \\
Cistocele grado I & $75 \%$ \\
Cistocele grado II & $15 \%$ \\
Cistocele grado III & $1 \%$ \\
\hline
\end{tabular}

La valoración del prolapso uterino la realizamos nosotros y si observamos que presenta prolapso uterino grado II o mayor, indicamos la colocación de malla Prolift total con conservación uterina.

\section{Procedimiento quirúrgico}

Se utilizó antibiótico profilaxis con cefazolina $2 \mathrm{~g}$ endovenoso una sola dosis una hora previa a la cirugía.

Las agujas para la colocación de la banda suburetral pueden pasar desde la cara interna del muslo hasta la región vaginal parauretral a través del agujero obturador y los músculos puborrectales o viceversa ${ }^{6}$. Se han descrito dos abordajes diferentes: desde el muslo hasta la vagina (técnica "out-in") y en dirección contraria ("in-out").

Cuando utilizamos la técnica in-out, (Sistema Gynecare TVT Obturador de Ethicon, Johnson \& Johnson), que fue en el 92\% de las TOT coloca$\operatorname{das}^{7,11}$.
Cuando utilizamos la técnica out-in (Monarc transobturator sling, American Medical System; Obtrix-Curved Boston Scientific) que fue en el 8\% de las TOT colocadas ${ }^{6}$.

Para ajustar la tensión de la malla en ambas técnicas la realizamos de la siguiente manera: cogimos un pellizco a la cinta de $2-3 \mathrm{~mm}$ con una pinza de allis, mientras se retira la envoltura plástica.

En los casos en que asoció otra cirugía ginecológica, se realizó en todos los casos en un tiempo posterior al TOT $^{7}$.

Aquellas pacientes que presentaban cistocele grado II o mayor en el mismo acto quirúrgico se realizó la corrección del cistocele con mallas de polipropileno Gynemesh (de Ethicon, Johnson \& Johnson) y en los casos en los que se observó colpocele se colocaron malla Prolift total (Ethicon, Johnson \& Johnson). La técnica quirúrgica es realizada por todos los miembros del Servicio.

De las 171 pacientes a 144 se les realizó TOT simple a 25 se les corrigió con malla de polipropileno el cistocele y a 2 se les coloco malla polipropileno total.

Todas las pacientes fueron intervenidas con anestesia general o raquídea.

Cuando realizamos TVT-O simple o asociado a corrección de cistocele ha habido una variación a lo largo de estos 2,5 años: En el año 2004 las pacientes a las que se les practicaba TVT-O se realizaba en régimen de Cirugía Mayor Ambulatoria y las pacientes eran dadas de alta sin sonda vesical. En el año 2005 debido a diferentes problemas que se presentaron en la Unidad de Cirugía Mayor Ambulatoria las pacientes eran ingresadas y se les mantenía la sonda vesical el día de la cirugia, problemas que se corrigieron en el 2006 por lo que volvimos a realizar la intervención en dicho régimen sin ingreso. Así de las 171 pacientes, 97 se realizaron en régimen de ambulatorio, 69 permanecieron 2 días ingresadas ( 1 día prequirúrgico, el día de la intervención y son dadas de alta al día siguiente) y 5 permanecieron más días ingresadas por diferentes complicaciones. Estas son las razones por las que existe una variabilidad en la cantidad de días de permanencia de sonda vesical en el postoperatorio inmediato así como en los días de ingreso. 
La evaluación post-quirúrgica incluyó datos sobre la cirugía, complicaciones intra y post-quirúrgica y los resultados de la cirugía. Las pacientes fueron valoradas a los tres, seis meses, al año y una vez al año, mediante historia clínica (valoración personal de su incontinencia y si requiere o no compresas) y exploración física que incluye valoración del cistocele, rectocele, colpocele y test de esfuerzo con vejiga llena.

\section{RESULTADOS}

Estimamos como curación objetiva la ausencia de pérdida de orina en la prueba de esfuerzo con vejiga llena y curación subjetiva aquella que refiere la paciente como ausencia de pérdida de orina $^{8}$. Por lo tanto, consideramos como curadas aquellas pacientes que tanto objetiva como subjetivamente no presentan escapes y no requieren compresas, y como fracaso cualquier grado de incontinencia aunque fuese menor que la previa a la cirugía.

Las pacientes fueron portadoras de sonda vesical una mediana de 0 días, media de 0,6 con un rango entre 0-9 días.

El seguimiento fue de 14 meses de mediana (rango 2-34 meses).

El fracaso de la técnica se produjo en el $13 \%, 15$ pacientes, apareciendo en todos los casos de forma precoz en el control posterior a la cirugía o en los 3 primeros meses.

Los fracasos se produjeron en 12 pacientes de las que sólo se les practicó intervención de TOT lo que supone el 7\% y 3 de las pacientes a las que se les realizó corrección de prolapsos asociados lo que supone el $11 \%$. De las pacientes en las que fracasó la técnica 11 presentaban preoperatoriamente IUE pura y 4 IU Mixta De los 15 fracasos a 3 pacientes no se les propuso nueva técnica quirúrgica ya que su incontinencia estaba asociada a urgencia con contracciones no inhibidas y a 2 se les coloco un REEMEX a otras 2 una TOT a otra Balones ACT y el resto no quieren realizarse absolutamente nada (Tabla 3).

Las complicaciones precoces ( ocurridas en las 48 horas posquirúrgicas) ocurrieron en el 7.7\%:1 perforación vesical, 1 hematoma en zona inguinal derecha que requirió reposo en cama en su domicilio, 3 con diferentes procesos médicos( broncoespasmo, crisis HTA, hipotensión), 1 hematoma
Tabla 3. Fracasos TOT

\begin{tabular}{llc}
\hline Fracaso por técnica quirúrgica & & \\
\hline TOT SIMPLE & 12 pacientes de 144 & $7 \%$ \\
TOT más Cirugía asociada & 3 pacientes de 27 & $11 \%$ \\
\hline Fracaso por tipo de incontinencia & \\
\hline Incontinencia de esfuerzo Pura & 11 pacientes de 110 & $10 \%$ \\
Incontinencia Mixta & 4 pacientes de 61 & $2,4 \%$ \\
\hline
\end{tabular}

en zona de Retzius- paravaginal izquierdo que requirió drenaje quirúrgico, 1 fiebre postoperatoria, 4 pacientes con sangrado vaginal y 2 con dolor en ambas zonas obturadoras, dolor de tal intensidad que era incapacitante. Todas las pacientes presentan dolor en ambas zonas inguinales que es de moderada intensidad y va despareciendo de forma gradual en 48-72 horas y no suele ser incapacitante (Tabla 4).

Tabla 4. Complicaciones precoces

\begin{tabular}{lcc}
\hline Perforación vesical & 1 paciente & $0,5 \%$ \\
Hematoma inguinal & 1 paciente & $0,5 \%$ \\
$\begin{array}{l}\text { Reintervención por hematoma en } \\
\text { espacio Retzius }\end{array}$ & 1 paciente & $0,5 \%$ \\
Fiebre & 1 paciente & $0,5 \%$ \\
Sangrado vaginal & 4 pacientes & $2 \%$ \\
Dolor Incapacitante & 2 pacientes & $1 \%$ \\
Procesos médicos & 3 pacientes & $1,7 \%$ \\
\hline
\end{tabular}

De nuestra serie el 35\% de las pacientes (61) presentaban incontinencia mixta aunque su componente fundamental era el esfuerzo. De éstas en 52 pacientes, el 85\%, la urgencia mejoró o desapareció y en el 15\% persiste. Estudiando el grupo de pacientes que persiste la urgencia, con historia clínica, exploración y urodinámica se observó que el $26 \%$ estaba asociado a obstrucción de la TOT, otro $26 \%$ estaba asociado a contracciones no inhibidas asociadas a volúmenes de 60 a 170 cc y en el resto su urodinámica era normal.

Como complicaciones tardías (aquellas aparecidas en el seguimiento) tenemos:

1. Retenciones post-operatorias (aquellas pacientes que requieren sonda vesical más de 24 horas y menos de 1 mes): está complicación se pre- 
sentó en 5 pacientes lo que supone un $3 \%$.Solo una llevó sonda durante 1 mes (urodinámica previa a intervención detrusor hipoactivo) que se solucionó. El resto fueron portadoras de sonda vesical durante 4 días.

2. Urgencia de novo: Apareció en el 2.3\% (excluidas de este porcentaje aquellas que su urgencia estaba asociada a obstrucción o que presentaban previamente urgencia) de las pacientes, y en estas se les asocia anticolinérgicos mejorando su cuadro clínico.

3. Dolor inguinal persistente (dolor que dura más de 1 mes): dolor en zona obturadora por donde salían las cintas. Se presenta en 3 pacientes $1,7 \%$.

4. Prolapso de órganos pélvicos: apareció en 2 pacientes a una de ellas se le había practicado una colocación de malla total prolift y se le realizó una histerectomía y a la otra se le había practicado corrección de cistocele y TOT, presentó prolapso uterino y se realizó histerectomía vaginal.

5. Dispareunia: 1 paciente.

6. Extrusión de la malla: Apareció en 5 pacientes. Se solucionó con extirpación de la malla y resutura vaginal evolucionando satisfactoriamente todas ellas.

7. Obstrucción al vaciamiento vesical: Esta complicación aparece entre los 4 y 12 meses de evolución. Su forma de presentación es variable: en el 60\% las pacientes presenta urgencia miccional y en el $40 \%$ aquejan dificultades miccionales. Para llegar al diagnóstico se les realiza urodinámica y ecografía postmiccional Esta complicación se presento en 9 pacientes (5,2\%). Se resolvió realizando corte lateral de la cinta. Todas menos una se mantienen continentes y la clínica ha desaparecido.

Globalmente las complicaciones afectan a un $16 \% . S i$ analizamos aquellas pacientes que se les practicó sólo TOT las complicaciones aparecen en el $14,4 \%$.

\section{DISCUSIÓN}

El procedimiento de la cinta vaginal sin tensión (TVT/TOT) para el tratamiento quirúrgico de la incontinencia de esfuerzo femenina, se basa en la teoría sobre los mecanismos del cierre uretral en las mujeres: la teoría integral descrita por Petros y Ulmsten en 1990, según la cual la ure- tra se cierra en su porción media y no en el cuello de la vejiga ${ }^{1,2}$. La falta de soporte de la uretra media por los ligamentos pubouretrales, de la pared vaginal anterior suburetral y el deterioro de la función y de la inserción de los músculos pubocccigeos predisponen a que las pacientes padezcan incontinencia de esfuerzo. Muchos autores sostienen que la TVT debe considerarse como gold estándar la corrección quirúrgica de la incontinencia de orina de esfuerzo, con cifras de hasta $89 \%$ de curación completa (Nuestra serie del $85 \%)^{10}$. Hoy por hoy las técnicas de cinta de polipropileno localizada en la uretra media, deben considerarse como la cirugía de elección en la IOE. El gold standard futuro será aquella cirugía que logre una continencia completa, sin morbilidad de importancia.

En el año 2001, Delorme et al describen la técnica de sling transobturador (TOT,) como una excelente alternativa al sling retropúbico, para reducir las complicaciones. La cinta es posicionada en forma holgada bajo la uretra media, extendiéndose en forma horizontal hacia los forámenes obturadores de la pelvis, disminuyendo el riesgo de obstrucción y retención urinaria por angulación excesiva de la uretra. Aun así, la literatura ha reportado casos de complicaciones y tasas de éxito similares a la TVT.

Un aspecto que consideramos importante, es si los buenos resultados obtenidos por el creador de la nueva técnica quirúrgica son obtenidos por otros grupos de trabajo y a este respecto observamos que los datos son reproducibles en todas las series, incluida la nuestra (Tabla 5) ${ }^{9,13,14,17-19}$.

Comparando los resultados de las TVT con los de las TOT realizados en nuestro servicio, observamos que los porcentajes de éxito de ambas técnicas en nuestras manos son muy similares aunque parece que los del abordaje transobturador son algo menores, si bien los periodos de seguimiento son diferentes. Otros autores que comparan ambas técnicas llegan a la misma conclusión, las tasas de éxito son similares (Tabla 6) ${ }^{12}$.

En cuanto a las complicaciones de la técnica, observamos que el tipo de complicaciones son diferentes entre ambas (tabla VII). La extrusión de la malla se presenta en un $2 \%$ de las TOT colocadas frente a un $0,3 \%$ de las TVT. Los porcentajes publicados de esta complicación en las series 
Tabla 5. Resultados de las diferentes series de TOT

\begin{tabular}{|c|c|c|c|c|}
\hline & $\mathbf{N}$ & Seguimiento & Éxito & Complicaciones \\
\hline Delorme E & 150 & 17 meses & $90 \%$ & $\begin{array}{l}\text { Obstrucción } 3 \% \\
\text { Urgencia de novo } 1,1 \%\end{array}$ \\
\hline Mellier G & 341 & 17 meses & $93 \%$ & Obstrucción 2\% \\
\hline Deval B & 129 & 17 meses & $89,9 \%$ & $\begin{array}{l}\text { Obstrucción 1,5\% } \\
\text { Erosión } 6,2 \%\end{array}$ \\
\hline Spinosa JP & 117 & 16 meses & $93 \%$ & Erosión 2,3\% \\
\hline Gunnemann A & 124 & 17 meses & $90 \%$ & \\
\hline Nuestra serie & 171 & 14 meses & $87 \%$ & $\begin{array}{l}\text { Extrusión } 2 \% \\
\text { Urgencia de novo } 1,1 \% \\
\text { Obstrucción } 4,8 \%\end{array}$ \\
\hline
\end{tabular}

Tabla 6. Tabla comparativa de resultados de nuestra serie TVT/TOT

\begin{tabular}{|c|c|c|c|c|}
\hline & $\begin{array}{l}\text { TVT } \\
\text { Seguimiento } \\
17 \text { meses } \\
\text { n } 114\end{array}$ & $\begin{array}{l}\text { TVT } \\
\text { Seguimiento } \\
35 \text { meses } \\
\text { n } 369\end{array}$ & $\begin{array}{l}\text { TOT } \\
\text { Seguimiento } \\
9 \text { meses } \\
\text { n } 117\end{array}$ & $\begin{array}{l}\text { TOT } \\
\text { Mediana } 14 \mathrm{~m} \\
\text { Media } 12 \\
\text { n } 171\end{array}$ \\
\hline Éxito & $93 \%$ & $85 \%$ & $84,7 \%$ & $87 \%$ \\
\hline IUE & $4 \%$ & $8,4 \%$ & $15,3 \%$ & $11,3 \%$ \\
\hline $\begin{array}{l}\text { Incontinencia } \\
\text { por urgencia }\end{array}$ & $3 \%$ & $5,8 \%$ & & $1,7 \%$ \\
\hline
\end{tabular}

Tabla 7. Tabla comparativa de complicaciones de nuestra serie TVT/TOT

\begin{tabular}{lccc}
\hline Complicaciones & TVT & TOT/malla & TOT \\
& N 369 & N 27 & N 144 \\
\hline Extrusión & $0,3 \%$ & $3,7 \%$ & $2 \%$ \\
Urgencia novo & $7 \%$ & $3,7 \%$ & $2,3 \%$ \\
Corte por Obstrucción & $5,7 \%$ & $7,5 \%$ & $4,8 \%$ \\
Hematomas & $4,6 \%$ & $0,0 \%$ & $1,3 \%$ \\
Sangrado vaginal & $0,3 \%$ & $7,5 \%$ & $1,3 \%$ \\
Retenciones & & & \\
postoperatorias & $17 \%$ & $6 \%$ & $2,7 \%$ \\
\hline
\end{tabular}

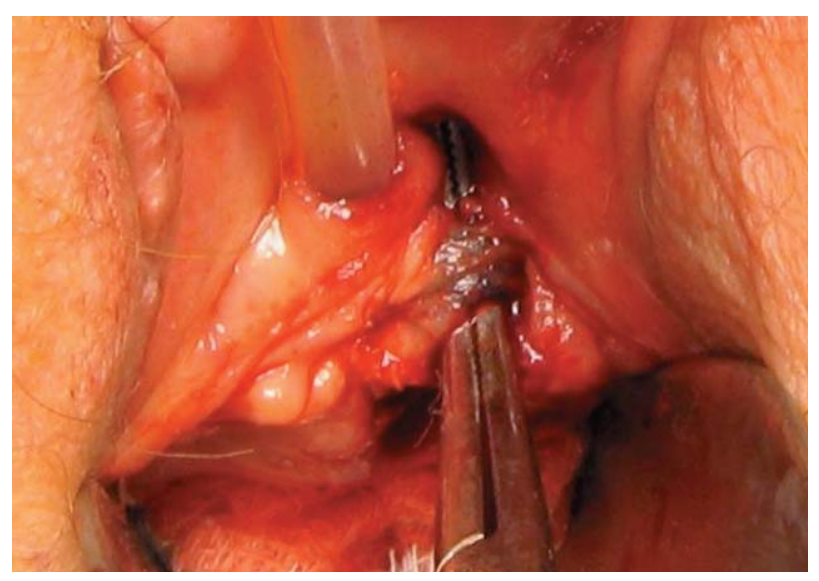

FIGURA 1. Corte lateral de cinta suburetral. de TOT, varían entre $0,07 \%$ hasta un $20 \%$ dependiendo del tipo de malla colocada ${ }^{13,14,16-19}$.

Respecto a la urgencia de novo en nuestra serie de TOT es de 2,3\% que es similar a todas las series publicadas y ésta es menor que con la TVT.

Respecto a la obstrucción es similar tanto en la TVT como en la TOT, unos porcentajes mayores que en el resto de publicaciones. Esto en principio lo atribuimos que buscamos la obstrucción a todas las pacientes que presentan urgencia miccional posquirúrgica. De hecho la mitad de las pacientes a las que cortamos la cinta presentaban urgencia con minimo residuo postmiccional y en la urodinámica se demostraba obstrucción. La resolución de esta complicación la realizamos de forma quirúrgica haciendo un corte lateral de la cinta. Con ello hemos conseguido que todas las pacientes menos una sigan continentes y la clínica de obstrucción desaparezca (Fig. 1).

Respecto a las retenciones urinarias postoperatorias(aquellas pacientes que requieren sonda vesical más de 24 horas y menos de 1 mes) es menor en la serie de TOT que en la de TVT. Comparar esta complicación con lo publicado en la literatura es difícil ya que la definición de la misma es diferente para cada autor.

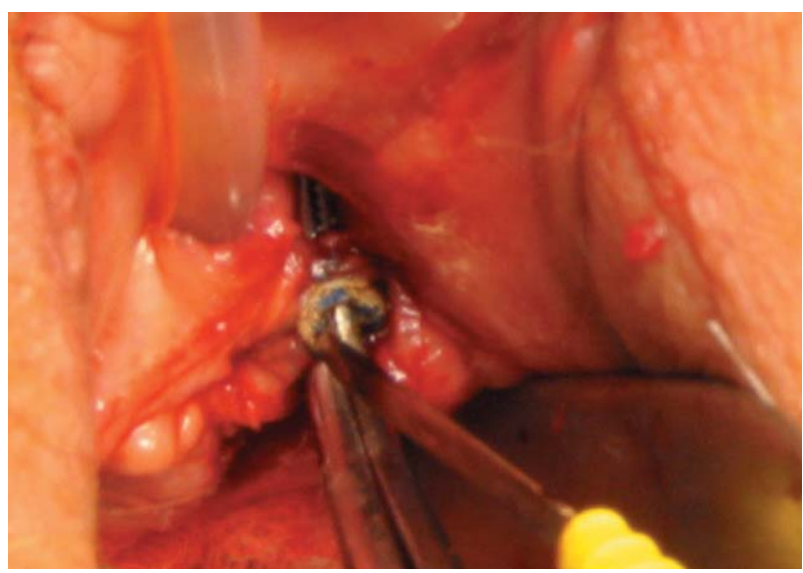


El resto de complicaciones son anecdóticas en nuestra serie de TOT, aunque todas ellas están descritas en la literatura.

Podemos concluir respecto a las complicaciones de la TOT respecto a la TVT que éstas son diferentes sus frecuencias y que la asociación de correcciones de cistoceles o prolapsos las aumentan.

\section{CONCLUSIONES}

La TOT es una técnica no exenta de complicaciones aunque la proporción de ellas es baja y con unos resultados que son alentadores.

Se trata de una técnica quirúrgica sencilla, que como toda técnica, tiene una curva de aprendizaje. Requiere un corto tiempo quirúrgico (menor que la TVT) y puede ser realizada en régimen de Cirugía Mayor Ambulatoria.

Las dos técnicas (TVT/TOT) han demostrado ser similares en cuanto a tasas de curación aunque en la serie de TOT es levemente más baja.

Las diferencias de las complicaciones se dan en los porcentajes aunque siempre escasas.

\section{REFERENCIAS}

1. Petros PE, Ulmsten UI. An integral theory of female urinary incontinence. Experimental and clinical considerations. Acta Obstet Gynecol Scand. 1990;153:7-31.

2. Petros PE, Ulmsten UI. An integral theory and its method for the diagnosis andmanagement of female urinary incontinence. Scand J Urol Nephrol. 1993;153:1-93.

3. Baden WF, Walker TA. Genesis of the vaginal profile: a corretale classification of the vaginal relaxation. Clin Obstet Gynecol. 1972;15(4):1048-1054.

4. Ulstem U, Petros P. Intravaginal slingplasty (IVS): an ambulatory surgical procedure for treatment of female urinary incontinence. Scand J Urol Nephrol. 1995;29(1):75-82.

5. Boublil V, Ciofu C, Traxer O, Sebe P, Haab F. Complications of urethral sling procedures. Curr Opin Obstet Gynecol. 2002; 14(5): 515-520.

6. Delorme E. Transobturator urethral suspension: mini-invasive procedure in the treatment of stress urinary incontinence in women. Prog Urol. 2001;11(6):1306-1313.

7. De Leval J. Novel surgical technique for the treatment of female stress urinary incontinence: transobturator vaginal tape inside-out. Eur Urol. 2003;44(6):724-730.

8. Meschia M, Pifarotti P, Bernasconi F, Guercio E, Maffiolini M, Magatti F, et al. Tension free vaginal tape: analysis of outcomes and complications in 404 stress incontinent women. International Urogynecology J Pelvic floor Dysfunct. 2001;12(Suppl. 2):S24-S27.
9. Debodinance P, Delporte P, Engrand JB, Boulogne M. Tension-free vaginal tape (TVT) in the treatment of urinary stress incontinence: 3 years experience involving 256 operations. Eur J Obstet Gynecol Reprod Biol. 2002;105(1): 49-58.

10. Jiménez Calvo J, Hualde Alfaro A, Santiago González de Garibay A, Pinós Paul M, Jiménez Aristu J, Montesino Semper M, et al. TVT: 3 años de experiencia. Actas Urol Esp. 2004;28(1):13-20.

11. Schanz Pardo J, Ricci Arriola P, Solà Dalenz V, Tacla Fernández X. Experiencia en la corrección quirúrgica de la incontinencia de orina de esfuerzo con técnica TOT (TransObturator-Tape). Arch Esp Urol. 2006;59(3):225-232.

12. Girvent Vilarnau, M; Cano Bachs S; Alvarez Cañadas V et al. Comparación entre las técnicas de TVT y TOT: efectividad y porcentaje de curación. e-Archivos de Ginecología y Obstetricia №8 2006.

13. Debodinance P. Trans-obturator urethral sling for surgical correction of female stress urinary incontinence: Outsidein (Monarc) versus inside-out (TVT-O). Are both ways safe?. J Gynecol Obstet Biol Reprod (Paris). 2006;35(6): 571-577.

14. Mellier G, Mistrangelo E, Gery L, Philippe C, Patrice M. Tension-free obturator tape (Monarc Subfascial Hammock) in patients with or without associated procedures. Int Urogynecol J Pelvic Floor Dysfunct. 2007;18(2):165-172.

15. Abdel-Fattah M, Sivanesan K, Ramsay I, Pringle S, Bjornsson S. How common are tape erosions? A comparison of two versions of the transobturator tension-free vaginal tape procedure. BJU Int. 2006;98(3):594-598.

16. Deval B, Haab F. Management of the complications of the synthetic slings. Curr Opin Urol. 2006;16(4):240-243.

17. Gunnemann A, Heleis W, Pohl J, Paliakoudis I, Thiel R. The transobturator tape (TOT). A minimally-invasive procedure for the treatment of female urinary stress incontinence. Urologe A. 2004;43(9):1106-1110.

18. Solà Dalenz V, Pardo Schanz J, Ricci Arriola P, Guiloff Fische E, Chiang Miranda H .Cirugía minimamente invasiva en la incontinencia urinaria femenina: TVT-O.V. Actas Urol Esp. 2006;30(1):61-66.

19. Hualde Alfaro A, Jiménez Calvo J, Sarmiento Gómez C, Pinos Paul M, de Pablo Cárdenas A, Ripa Saldias L, et al. Nuestra experiencia a cinco años y medio en TVT. Actas Urol Esp. 2006;30(2):181-185.

Correspondencia autor: Dr. J. Jiménez Calvo Servicio de Urología. Hospital Virgen del Camino Irunlarrea 4 - 31009 Pamplona (Navarra) Tel.: 948429400

E-mail autor: auvc03@yahoo.es

Información artículo: Original- Incontinencia urinaria femenina Trabajo recibido: octubre 2006

Trabajo aceptado: septiembre 2007 